POS $\quad$ PROCEEDINGS

\title{
Investigating the high-energy emission from Centaurus A and XTE J1550-564
}

Valentina Bianchin*

INAF - IASF Bologna, Italy

E-mail: bianchineiasfbo.inaf.it

\section{Luigi Foschini}

INAF-IASF Bologna, Italy

\section{Rita Sambruna}

$N A S A / G S F C, U S A$

\section{Guido Di Cocco}

INAF-IASF Bologna, Italy

\section{Giuseppe Malaguti}

INAF-IASF Bologna, Italy

\section{Fulvio Gianotti}

INAF-IASF Bologna, Italy

\section{Filomena Schiavone}

INAF-IASF Bologna, Italy

\begin{abstract}
A preliminary comparative study of the $\mathrm{X} / \gamma$-ray emission of the radio galaxy Cen $\mathrm{A}$ and the microquasar XTE J1550-564 is presented. The similar orientation of the jet in the galactic and extragalactic object, by minimizing the geometrical effects, allow searching for intrinsic physical similarities/differences between the two sources.
\end{abstract}

VI Microquasar Workshop: Microquasars and Beyond September 18-22, 2006

Como, Italy

\footnotetext{
* Speaker.
} 


\section{Introduction}

Despite the large scale difference, microquasars and radio loud active galactic nuclei are thought to be powered essentially by the same mechanisms: a black hole surrounded by an accretion disc, a corona and a bipolar relativistic jet. In order to investigate the differences and similarities of the processes at work in both kinds of objects we have analyzed the high energy emission from the extragalactic source Centaurus A and the microquasar XTE J1550-564, since their similar orientation with respect to the line of sight minimizes the geometrical effects.

Centaurus A is a FRI radio galaxy at $\sim 3.8 \mathrm{Mpc}$, and VLBI observations indicate the presence of a relativistic jet inclined by $50^{\circ}-80^{\circ}$ ([9]). This source has been observed in the hard Xray band by different missions such as BeppoSAX, CGRO, RXTE, revealing that the continuum is characterized by a strongly absorbed $\left(N_{H} \sim 10^{23} \mathrm{~cm}^{-2}\right)$ power-law with $\Gamma \sim 1.8$ (see e.g. [5]).

XTE J1550-564 is a Low Mass X-ray binary at a distance of $\sim 5.3 \mathrm{kpc}$, hosting a black hole with mass $M=8.36-10.76 M_{\odot}$ ([6]). After the major outburst in 1998, observations of the radio flares have revealed a relativistic mass ejection with inclination $70.8^{\circ} \div 75.4^{\circ}$ ([6]).

Both sources have been observed several times by the INTEGRAL satellite ([12]), and the galactic object, in particular, has been detected at high energies also by the IBIS/PICsIT instrument ([2], [11]). In the following sections we present the analysis of all the publicly available INTEGRAL data in order to search for differences/similarities of the high energy emission.

\section{Data selection}

For both the extragalactic and the galactic source we extracted all the available INTEGRAL data from the public archive ${ }^{1}$. Among all INTEGRAL observations of Cen A we selected the pointings with the source in the IBIS fully coded FOV (within $5^{\circ}$ from the instrument boresight). These correspond to revolutions 48, 49, 93, 149, 150, 163, covering a period from 7 March 2003 to 14 February 2004. The analysis of JEMX, SPI, IBIS/ISGRI together with Rossi X-Ray Timing Explorer for all observations but Revs. 93 and 150, has been already presented in [7]. Here we reanalyze data collected by the IBIS instrument, including the two mentioned above.

XTE J1550-564 was regularly observed by INTEGRAL during the Galactic Plane Scan of the Core Programme. On March 2003, during revolution 54, INTEGRAL observed the source in outburst [3] and triggered a TOO activated during the following days (revolutions 55, 57, 58, 60). The IBIS/ISGRI, JEM-X and SPI data of this outburst were already presented in [8], where the authors noted that the source remained in a hard spectral state $(\Gamma \sim 1.5)$ during the outburst phase followed by INTEGRAL: they concluded that the source had a failed outburst and never switched to the soft state. Here we present a re-analysis of the IBIS/ISGRI and, for the first time, PICsIT data, covering an energy range up to $\sim 0.7 \mathrm{MeV}$. INTEGRAL data of the outburst refers to the period from 27 March to 13 April 2003, for a total exposure of $\sim 200 \mathrm{ks}$. In addition we have also collected and analyzed all the INTEGRAL public pointings after the outburst where XTE J1550-564 was inside the fully coded FOV.

Since simultaneous RXTE observations of the Cen A and of the outburst of XTE J1550-564 are available in the public archive, we include these data in the broad band spectral analysis: for

\footnotetext{
${ }^{1}$ http://isdc.unige.ch/index.cgi?Data+browse
} 

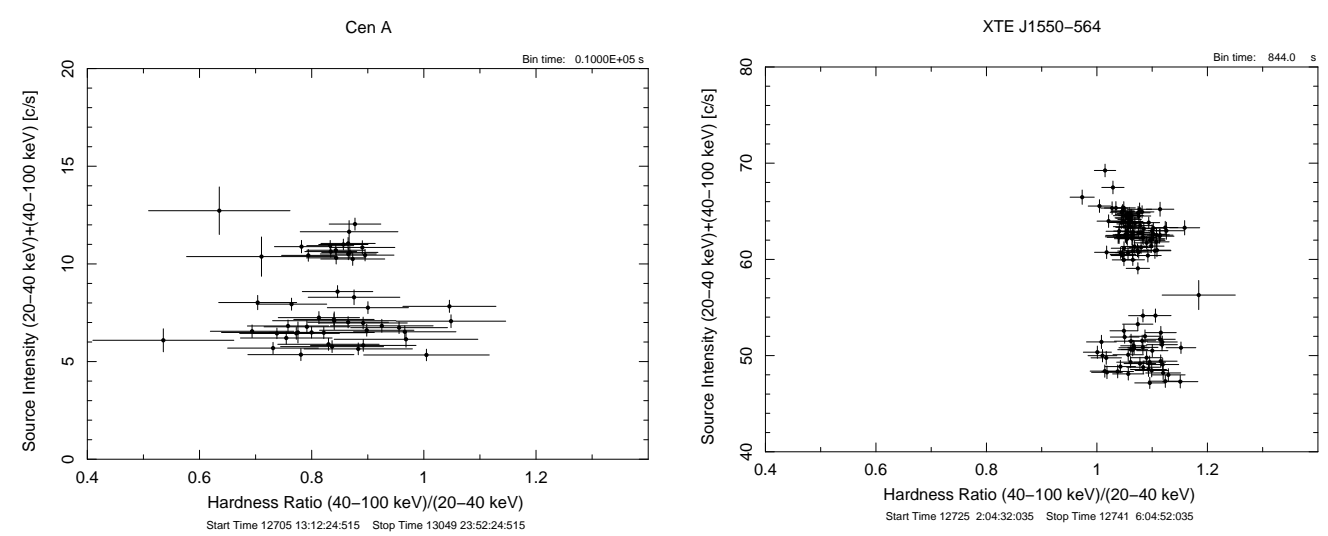

Figure 1: Hardness-intensity diagram for Cen A (left) and XTE J1550-564 (right). The hardness ratio is defined as the flux in the $40-100 \mathrm{keV}$ band divided by the flux in the $20-40 \mathrm{keV}$.

this purpose we selected the standard products (stdprd2) of the RXTE/PCA instrument. Cen A was observed by RXTE from 7 March 2003 to 14 February 2004, with an exposure of $\sim 80$ ks during Revs. 48+49, $\sim 90$ ks during Rev. 149 and $\sim 40$ ks during Rev. 163. XTE J1550-564 was observed from 23 March to 12 April 2003, for a total exposure of $\sim 70 \mathrm{ks}(\sim 5 \mathrm{ks}$ during Rev. 55, $\sim 24 \mathrm{ks}$ during Revs. 57+58 and $\sim 41$ ks during Rev. 60)

\section{Data Analysis and Results}

INTEGRAL data analysis has been carried out by means of the software package OSA $5.1^{2}$. For both sources we have produced lightcurves using IBIS/ISGRI data in $20-40 \mathrm{keV}$ and $40-$ $100 \mathrm{keV}$ energy bands and the hardness-intensity diagram, where we define the hardness ratio as the flux in $40-100 \mathrm{keV}$ divided by the flux in the $20-40 \mathrm{keV}$ band (Fig.1). INTEGRAL data for Cen A show a flux decrease, in both energy bands, by a factor $\sim 2$ from Revs. $48-93$ to Rev. 149 and a subsequent slow increase during Rev. 163. We searched for a detection with IBIS/PICsIT in the mosaic of all the observations (Revs. 48-163, total exposure of $\sim 590 \mathrm{ks}$ ) but with negative results. An upper limit $(3 \sigma)$ of $2.3 \times 10^{-10} \mathrm{erg} \mathrm{cm}^{-2} \mathrm{~s}^{-1}$ was set in the $252-336 \mathrm{keV}$ energy band.

For XTE J1550-564 we have analyzed all the INTEGRAL/ISGRI pointings after the outburst when the source was in the fully coded FOV. In the mosaic of all the observations, with a total exposure of $\sim 690 \mathrm{ks}$, no detection was found and with an upper limit $(3 \sigma)$ of $0.7 \mathrm{mCrab}$ in the $20-40 \mathrm{keV}$ energy band, corresponding to a source luminosity $<1.6 \times 10^{34} \mathrm{erg} \mathrm{s}^{-1}$, in agreement with the extrapolation from [1] during the quiescent phase. During the outburst the microquasar increased its flux, in both energy bands, of about two orders of magnitude with respect to the flux values in quiescence. Despite the flux changes, both sources remained in a hard spectral state, with Cen A showing lower hardness with respect to the galactic source.

For both sources we have performed a broad band spectral analysis using PCA, IBIS/ISGRI and, for XTE J1550-564, IBIS/PICsIT data. PICsIT spectral data and errors have been derived from intensity and significance maps on the 8 standard energy bands available in OSA for single events.

\footnotetext{
${ }^{2}$ http://isdc.unige.ch/?Soft+download
} 

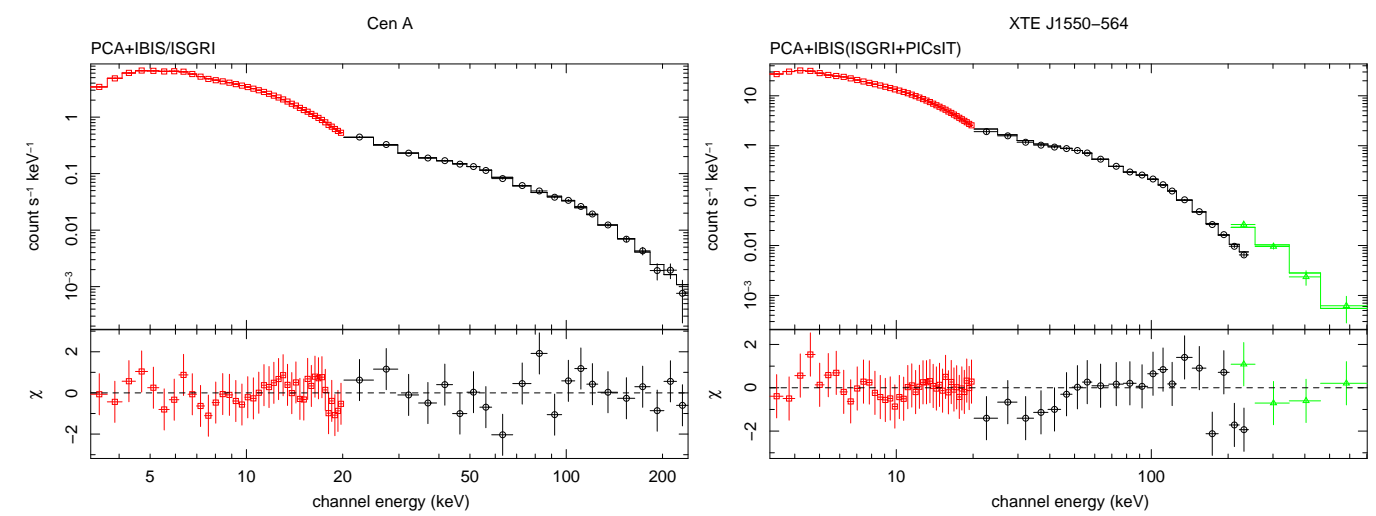

Figure 2: Joint fit spectral analysis for Cen A (left panel) during Revs. 48+49+93, and XTE J1550-564 (right panel) on the overall outburst (Revs. 55-60). The lower panels show the residuals in $\sigma$ units.

\begin{tabular}{|c|c|c|c|c|c|c|c|c|c|c|c|}
\hline $\begin{array}{l}\text { Rev } \\
\text { (1) }\end{array}$ & $\begin{array}{l}N_{\mathrm{H}} \\
(2) \\
\end{array}$ & $\begin{array}{c}\Gamma \\
\text { (3) } \\
\end{array}$ & $\begin{array}{c}N \\
\text { (4) }\end{array}$ & $\begin{array}{c}R \\
(5) \\
\end{array}$ & $\begin{array}{l}E_{\mathrm{c}} \\
(6)\end{array}$ & $\begin{array}{c}E_{\mathrm{Fe}} \\
(7) \\
\end{array}$ & $\begin{array}{c}E q . W \\
(8)\end{array}$ & $\begin{array}{c}F_{\text {line }} \\
(9)\end{array}$ & $\begin{array}{c}\chi_{r}^{2} \\
(10)\end{array}$ & $\begin{array}{c}F_{20-40 \mathrm{keV}} \\
\text { (11) }\end{array}$ & $\begin{array}{c}F_{5-500 \mathrm{keV}} \\
(12)\end{array}$ \\
\hline \multicolumn{12}{|c|}{ Centaurus A } \\
\hline $48+49+93$ & $15.3_{-0.4}^{+0.4}$ & $1.84_{-0.02}^{+0.02}$ & $0.21_{-0.01}^{+0.01}$ & $0.13_{-0.07}^{+0.07}$ & $600 f$ & $6.23_{-0.12}^{+0.17}$ & $71_{-19}^{+18}$ & $0.56_{-0.15}^{+0.13}$ & 0.55 & 3.89 & 2.36 \\
\hline $149+150$ & $17.8_{-0.5}^{+0.5}$ & $1.85_{-0.03}^{+0.01}$ & $0.12_{-0.01}^{+0.01}$ & $<0.17$ & $600 f$ & $6.40_{-0.18}^{+0.09}$ & $128_{-19}^{+27}$ & $0.52_{-0.09}^{+0.10}$ & 0.79 & 2.16 & 1.31 \\
\hline 163 & $16.2_{-0.5}^{+0.5}$ & $\begin{array}{l}1.83_{-0.03}^{+0.03} \\
\end{array}$ & $0.13_{-0.01}^{+0.01}$ & $0.13_{-0.10}^{+0.11}$ & $600 f$ & $\begin{array}{l}6.36_{-0.12}^{+0.03} \\
\end{array}$ & $128_{-31}^{+71}$ & $\begin{array}{l}0.53_{-0.10}^{+0.10} \\
\end{array}$ & 0.84 & 2.57 & 1.57 \\
\hline \multicolumn{12}{|c|}{ XTE J1550-564 } \\
\hline 55 & $0.85 f$ & $1.46_{-0.02}^{+0.02}$ & $0.20_{-0.01}^{+0.01}$ & $0.69_{-0.10}^{+0.10}$ & $278_{-30}^{+37}$ & $6.4 f$ & $88_{-18}^{+19}$ & $1.23_{-0.25}^{+0.26}$ & 0.52 & 16.55 & 10.85 \\
\hline $57+58$ & $0.85 f$ & $1.50_{-0.02}^{+0.02}$ & $0.28_{-0.01}^{+0.01}$ & $0.87_{-0.10}^{+0.10}$ & $298_{-33}^{+41}$ & $6.4 f$ & $78_{-18}^{+18}$ & $1.39_{-0.33}^{+0.35}$ & 0.57 & 21.16 & 13.34 \\
\hline 60 & $0.85 f$ & $1.52_{-0.02}^{+0.02}$ & $0.31_{-0.01}^{+0.01}$ & $0.87_{-0.10}^{+0.11}$ & $271_{-27}^{+34}$ & $6.4 f$ & $70_{-17}^{+18}$ & $1.37_{-0.36}^{+0.33}$ & 0.58 & 22.05 & 13.30 \\
\hline TOT & $0.85 f$ & $1.52_{-0.02}^{+0.02}$ & $0.29_{-0.01}^{+0.01}$ & $0.83_{-0.10}^{+0.10}$ & $292_{-27}^{+31}$ & $6.4 f$ & $72_{-18}^{+17}$ & $1.29_{-0.32}^{+0.36}$ & 0.55 & 20.25 & 12.57 \\
\hline
\end{tabular}

Table 1: Summary of fits with the XSPEC model wabs * (pexrav+gauss). Columns: (1) Revolution group; (2) Column density [10 ${ }^{22} \mathrm{~cm}^{-2}$ ]; (3) Photon index; (4) Normalization at $1 \mathrm{keV}$ [ph keV $\mathrm{kem}^{-1} \mathrm{~cm}^{-1}$ ]; (5) Reflection parameter; (6) Cut-off energy [keV]; (7) Line centroid [keV]; (8) Line equivalent width [eV]; (9) Line flux $\left[10^{-3} \mathrm{ph} \mathrm{cm}^{-2} \mathrm{~s}^{-1}\right]$; (10) Reduced $\chi^{2}$ (Cen A: 54 dof; XTE J1550-564: 55 dof and 59 dof in TOT group); (11) Model flux in $20-40 \mathrm{keV}$ $\left[10^{-10} \mathrm{erg} \mathrm{cm}^{-2} \mathrm{~s}^{-1}\right]$; (12) Model flux in $5-500 \mathrm{keV}\left[10^{-9} \mathrm{erg} \mathrm{cm}^{-2} \mathrm{~s}^{-1}\right] . f$ indicates fixed parameter.

ISGRI spectra are obtained with a response matrix rebinned in order to have the best sampling in the $20-250 \mathrm{keV}$ range. ISGRI spectra are combined using the spe_pick tool included in the OSA 5.1 while PCA averaged spectrum and response matrix are obtained respectively by matpha and addrmf task included in FTOOLS V.6.1.1. A systematic error of $1 \%$ has been added to ISGRI and PCA combined spectra and $1 \%$ systematic error is considered in the first energy bin of PICsIT spectrum. Spectral analysis has been carried out by means of xspec v.11.

After a preliminary analysis for each individual revolution on ISGRI and PCA data separately, all observations have been grouped on longer periods, since hardness versus intensity diagrams (Fig.1) have shown no significant spectral change during flux variations. After having explored several models, we found that the best fit is obtained with the model wabs * (pexrav+ gauss) for both sources. The results of the global fit are summarized in Table 1.

For Cen A the high energy cutoff is frozen at $600 \mathrm{keV}$ (see [5]) and an unresolved line is considered. We fixed the source orientation at the same value of the galactic object $\left(72.6^{\circ}\right)$. We found (Table 1) that the photon index remains constant while the normalization significantly reduces after the first group of observations. The reflection contribution turned out to be almost negligible. Nevertheless when considering a power-law (with high energy cutoff), we obtain the same values (within errors) for the parameters describing the line and the incident power-law spectrum while the goodness of the fit is not improved. The iron line does not change significantly neither in posi- 
tion nor in flux, although the decrease in the continuum. This seems to confirm that the iron line is not due to reflection.

For XTE J1550-564 we found that the best fit model is still an absorbed powerlaw with a reflection component and a gaussian line (Table 1). We check the accordance with an ionized reflection model but we found an ionization parameter $<10^{-3}$. The column density is fixed at $N_{H}=0.85 \times 10^{22} \mathrm{~cm}^{-2}([10])$ and the line is frozen at $6.4 \mathrm{keV}$ with unresolved width. Comparing our results with [8], we recognize a slightly steepening of the power-law as the source moves to higher flux level. We found a larger reflection component, increasing with the flux level, and a cut-off occurring at lower energies. Furthermore the availability of PICsIT data allowed us to have signal up to $\sim 700 \mathrm{keV}$ (Fig.2) with no evidence for a hard tail (see [4]).

\section{Conclusions}

We have analyzed all the publicly available INTEGRAL observations of Cen A and XTE J1550-564. The present data set does not allow to find significant differences or similarities between the extragalactic source Cen A and the galactic microquasar XTE J1550-564. Both sources remained hard during flux changes, however the spectra suggest that different mechanisms are at work in the two sources.

\section{References}

[1] Corbel S., Tomsick J., Kaaret P., 2006, On the origin of Black Hole X-Ray emission in quiescence: Chandra observations of XTE J1550-564 and H1743-322, APJ, 636, 971

[2] Di Cocco G., Caroli E., Celesti E. et al., 2003, IBIS/PICsIT in flight performances, A\&A, 411, L189

[3] Dubath P., Revnivtsev M., Goldoni P. et al., 2003, XTE J1550-564, IAUC, 8100, 1

[4] Gierliǹski M. \& Done C., The X-ray/ $\gamma$-ray spectrum of XTE J1550-564 in the very high state, 2003, MNRAS, 342, 1083

[5] Grandi P., Fiocchi M., Perola G. C., et al., 2003, BeppoSAX observations of Centaurus A: the hard continuum and the iron line feature, ApJ, 593, 160

[6] Orosz J. A., Groot P. J., van der Klis M. et al., 2002, Dynamical Evidence for a Black Hole in the Microquasar XTE J1550-564, ApJ, 568, 845

[7] Rothschild R. E., Wilms J., Tomsick J. A., et al., 2006, INTEGRAL and RXTE observations of Centaurus A, ApJ, 641, 801

[8] Sturner S. J. \& Shrader C. R., 2005, XTE J1550-564: INTEGRAL observations of a failed outburst, ApJ, 625, 923

[9] Tingay S. J., Jauncey D. L., Reynolds J. E. et al., 1998, The subparsec-scale structure and evolution of Centaurus A: the Nearest Active Radio Galaxy, ApJ, 115, 960

[10] Tomsick J. A., Corbel S., Kaaret P>, 2001, X-Ray Observations of XTE J1550-564 during the Decay of the 2000 Outburst. I. Chandra and RXTE Energy Spectra, ApJ, 563, 229

[11] Ubertini P., Lebrun F., Di Cocco G., et al., 2003, IBIS: the Imager on-board INTEGRAL, A\&A, 411, L131

[12] Winkler C., Courvoisier T. J. L., Di Cocco G. et al., 2003, The INTEGRAL mission, A\&A, 411, L1 